\title{
BROWDER SPECTRAL SYSTEMS
}

\author{
RAÚL E. CURTO AND A. T. DASH
}

(Communicated by Paul S. Muhly)

\begin{abstract}
For two spectral systems $\sigma_{1}$ and $\sigma_{2}$ on a Banach space $\mathscr{Z}$, the associated Browder spectral system is $\sigma_{b ; 1,2}:=\sigma_{1} \cup \sigma_{2}^{\prime}$. We prove that $\sigma_{b ; 1,2}$ possesses the projection and spectral mapping properties whenever $\sigma_{1}$ and $\sigma_{2}$ do (and satisfy a few additional mild assumptions). We also calculate $\sigma_{b ; 1,2}$ for tensor products. The results extend several previous works on Browder spectra.
\end{abstract}

1. Introduction. The Browder spectrum of an operator $T$ acting on a Banach space $\mathscr{X}$ is usually defined as

$$
\sigma_{b}(T):=\sigma_{e}(T) \cup \sigma(T)^{\prime}
$$

the union of the essential spectrum and the limit points of the spectrum. B Gramsch and D. Lay showed in [9] that

$$
\sigma_{b}(f(T))=f\left(\sigma_{b}(T)\right)
$$

for every function $f$ analytic on a neighborhood of $\sigma(T)$. Browder spectra have been considered by many authors; we only mention here the works $[1,6.13$, and 14], which deal with various notions of joint Browder spectra. We can encompass these notions in a very general one involving arbitrary spectral systems. Roughly speaking, a spectral system on $\mathscr{X}$ assigns a compact nonempty subset of $\mathbf{C}^{n}$ to every commuting $n$-tuple of operators on $\mathscr{X}$ (the sizes of the tuples are allowed to vary). Given two spectral systems $\sigma_{1}$ and $\sigma_{2}$, the Browder spectral system associated with $\sigma_{1}$ and $\sigma_{2}$ is

$$
\sigma_{b ; 1,2}:=\sigma_{1} \cup \sigma_{2}^{\prime},
$$

where ' stands for the set of limits points. We first obtain conditions for $\sigma_{b ; 1,2}$ to possess the projection property and we subsequently proceed to consider the spectral mapping property via the functional representation obtained by W. Żelazko in [18] (see also [4, $\S \S 2$ and 3]). Finally, we calculate $\sigma_{b ; 1,2}$ in the case of tensor products for suitable choices of $\sigma_{1}$ and $\sigma_{2}$, particularly $\sigma_{1}=\sigma_{T e}$ and $\sigma_{2}=\sigma_{T}$, the Taylor essential and Taylor spectra, respectively. Our main result, Theorem

Received by the editors February 17, 1987 and, in revised form, May 4, 1987.

1980 Mathematics Subject Classification (1985 Revision). Primary 47A10, 47A53, 47A50; Secondary 47A62.

Key words and phrases. Spectral system, Browder joint spectra, projection property, tensor products.

Research of the first author partially supported by a grant from NSF.

Research of the second author partially supported by a grant from NSERC. 
2.8 , establishes that whenever $\sigma_{1}$ and $\sigma_{2}$ possess the projection property and every isolated point of $\sigma_{2}(S)$ is isolated in $\sigma_{T}(S)$, we have

$$
P_{S} \sigma_{b ; 1,2}(S, T)=\sigma_{b ; 1,2}(S)
$$

for all $T$ such that $(S, T)$ is commuting. (Here $S$ and $T$ are tuples and $P_{S}$ projects onto the $S$-coordinates; to be precise, a few extra assumptions are needed, but they are quite mild and satisfied by most known spectral systems.) Once we know that $\sigma_{b ; 1,2}$ possesses the projection property, we can establish a spectral mapping theorem for (vector-valued) functions analytic in neighborhoods of the Taylor spectrum, extending to $n$-variables some of Gramsch and Lay's results. Theorem 2.8 also provides a good source of spectral systems possessing the projection property; as is known, such systems generally possess the spectral mapping property for polynomial mappings (see $\S 3$ below).

2. The projection property for Browder spectral systems. For $\mathscr{X}$ a Banach space, we let $\mathscr{L}(\mathscr{X})$ denote the algebra of all (bounded) operators on $\mathscr{X}: \mathscr{L}(\mathscr{X})_{\text {com }}^{(n)}$ will denote the collection of all commuting $n$-tuples of elements in $\mathscr{L}(\mathscr{X})$. Following Żelazko [18], we write $S \subset \mathscr{L}(\mathscr{X})$ if $S \in \mathscr{L}(\mathscr{X})_{\text {com }}^{(n)}$ for some $n \geq 1$. A spectral system $\tilde{\sigma}$ on $\mathscr{X}$ is an assignment $S \mapsto \tilde{\sigma}(S)$ defined on $\bigcup_{n \geq 1} \mathscr{L}(\mathscr{X})_{\text {com }}^{(n)}$ and such that $\tilde{\sigma}(S)$ is a compact nonempty subset of $\mathbf{C}^{n}$ whenever $S \in \mathscr{L}(\mathscr{X})_{\text {com }}^{(n)}$. A spectral system possesses the projection property if

$$
P_{S} \tilde{\sigma}(S, T)=\tilde{\sigma}(S) \quad \text { and } \quad P_{T} \tilde{\sigma}(S, T)=\tilde{\sigma}(T)
$$

for all $(S, T) \subset \mathscr{L}(\mathscr{Z})$. (Here $P_{S}$ and $P_{T}$ are the canonical projections onto the coordinates of $S$ and $T$, respectively.)

Spectral systems possessing the projection property have a privileged position among general spectral systems: Under the assumption $\tilde{\sigma} \subseteq \hat{\sigma}$ (the polynomially convex spectrum), such a $\tilde{\sigma}$ will also possess the spectral mapping theorem for functions analytic in a neighborhood of $\hat{\sigma}$. (A more general result is true, but we will not try to describe it here; see $[4,11]$.) Our aim is to obtain the projection property for Browder spectral systems and then to apply the above result to give very general spectral mapping theorems for Browder spectra, extending greatly on previous work of B. Gramsch and D. Lay [9].

DEFINITION 2.1. Let $\mathscr{X}$ be a Banach space and let $\sigma_{1}$ and $\sigma_{2}$ be two spectral systems on $\mathscr{X}$. The Browder spectral system associated with $\sigma_{1}$ and $\sigma_{2}$ is

$$
\sigma_{b ; 1,2}=\sigma_{1} \cup \sigma_{2}^{\prime},
$$

where ' stands for the set of limit points. It goes without saying that $\sigma_{b ; 1,2}$ is indeed a spectral system. Also, if $\sigma_{1}=\sigma_{T e}$ and $\sigma_{2}=\sigma_{T}$ (the Taylor essential and Taylor spectra, respectively, $[\mathbf{4}, \mathbf{1 5}])$, and $S$ is an operator on $\mathscr{X}$, then $\sigma_{b ; 1,2}(S)=\sigma_{b}(S)$, the usual Browder spectrum.

PROPOSITION 2.2. Let $\sigma_{1}$ and $\sigma_{2}$ be spectral systems for $\mathscr{L}(\mathscr{X})$, and let $\sigma_{b ; 1,2}$ be the Browder spectral system associated to $\sigma_{1}$ and $\sigma_{2}$. Assume that $\sigma_{1}$ and $\sigma_{2}$ possess the projection property. For $(S, T) \subset \mathscr{L}(\mathscr{X})$ we then have

$$
\sigma_{b ; 1,2}(S) \subseteq P_{S} \sigma_{b ; 1,2}(S, T) .
$$

Proof. Let $\lambda \in \sigma_{b ; 1,2}(S)$. If $\lambda \in \sigma_{1}(S)$, then there exists $\mu \in \sigma_{1}(T)$ such that $(\lambda, \mu) \in \sigma_{1}(S, T) \subseteq \sigma_{b ; 1,2}(S, T)$, and therefore $\lambda \in P_{S} \sigma_{b ; 1,2}(S, T)$. If $\lambda \in$ $\sigma_{2}(S)^{\prime}$ instead, then $\lambda=\lim _{n} \lambda_{n}$, where $\left\{\lambda_{n}\right\}$ is a sequence of distinct points of 
$\sigma_{2}(S)$. By the projection property for $\sigma_{2}$, we can then find $\mu_{n} \in \sigma_{2}(T)$ such that $\left(\lambda_{n}, \mu_{n}\right) \in \sigma_{2}(S, T)$. By the compactness of $\sigma_{2}(T)$, there exists a subsequence $\mu_{n_{k}} \rightarrow \mu \in \sigma_{2}(T)$. Thus, $(\lambda, \mu)$ is the limit of a sequence of distinct points of $\sigma_{2}(S, T)$, so $\lambda \in P_{S} \sigma_{b ; 1,2}(S, T)$.

REMARK 2.3. The containment in Proposition 2.2 is usually the harder part in a proof of the projection property, since most spectral systems $\tilde{\sigma}$ satisfy $\tilde{\sigma}(S, T) \subseteq$ $\tilde{\sigma}(S) \times \tilde{\sigma}(T)$; this is not the case here, as the following example shows. As usual, $\sigma_{l}$ and $\sigma_{r}$ denote the left and right spectra, respectively.

EXAMPLE 2.4. Let $\mathscr{H}$ be a Hilbert space and let $S \subset \mathscr{L}(\mathscr{X})$ be such that $0 \notin \sigma_{r}(S)$ and $\sigma_{l}(S)=\{0\} \dot{\cup} K$, where $K$ is compact and nonempty (one such $S$ was constructed in [8]; see also [3]), and let $T$ be an operator on $\mathscr{H}$ such that $0 \in \sigma_{l}(T)^{\prime}$. Consider $(S \otimes I, I \otimes T)$. By [10],

$$
\sigma_{l}(S \otimes I, I \otimes T)=\sigma_{l}(S) \times \sigma_{l}(T)=\left[\{0\} \times \sigma_{l}(T)\right] \dot{\cup}\left[K \times \sigma_{l}(T)\right] .
$$

It follows that $0 \in \sigma_{l}(S \otimes I, I \otimes T)^{\prime}$, while $0 \notin \sigma_{l}(S)^{\prime}$. If we let $\sigma_{1}:=\sigma_{r}$ and $\sigma_{2}:=\sigma_{l}$, we see that

$$
\sigma_{b ; 1,2}(S \otimes I, I \otimes T) \nsubseteq \sigma_{b ; 1,2}(S \otimes I) \times \sigma_{b ; 1,2}(I \otimes T) .
$$

The above example indicates that additional assumptions on $\sigma_{2}$ must be made to obtain the reverse inclusion for the projection property. For a Banach space $\mathscr{X}$ we shall let $\mathscr{S}_{c ; \infty}(\mathscr{X})$ denote the collection of infinite dimensional subspaces of $\mathscr{X}$ that admit a Banach space complement, i.e., $\mathscr{M} \in \mathscr{S}_{c ; \infty}(\mathscr{X})$ if and only if $\operatorname{dim} \mathscr{M}=\infty$ and there exists a subspace $\mathscr{N}$ such that $\mathscr{X}=\mathscr{M}+\mathscr{N}$. Of course, if $\mathscr{X}$ is infinite dimensional, $\mathscr{X} \in \mathscr{S}_{c ; \infty}(\mathscr{X})$.

DEFINITION 2.5. Let $\tilde{\sigma}=\left\{\tilde{\sigma}_{\mathscr{M}}\right\}_{\mathscr{M} \in \mathscr{S}_{c ; \infty}(\mathscr{X})}$ be a family of spectral systems $\left(\tilde{\sigma}_{\mathscr{M}}\right.$ acting on $\mathscr{M})$. We shall say that $\tilde{\sigma}$ is monotone if for all $\mathscr{M} \in \mathscr{S}_{c ; \infty}(\mathscr{X})$ and all $T \subset \mathscr{L}(\mathscr{X})$ such that $T \mathscr{M} \subseteq \mathscr{M}$ and $T \mathscr{N} \subseteq \mathscr{N}$ (where $\mathscr{M} \dot{+N}=\mathscr{X}$ ), one has $\tilde{\sigma}_{\mathscr{M}}\left(\left.T\right|_{\mathscr{M}}\right) \subseteq \tilde{\sigma}_{\mathscr{X}}(T)$. Most known spectral systems give rise to montone families. For instance, spatial systems $\left(\sigma_{l}, \sigma_{r}, \sigma_{T}\right.$, Słodkowski's joint spectra [13]), their essential counterparts $\left(\sigma_{l e}, \sigma_{e}\right.$, etc. $)$, and the product spectrum $\left.\sigma_{\Pi}:=\sigma \times \sigma \times \cdots \times \sigma\right)$ are monotone. Moreover, a calculation shows that the commutant and bicommutant spectra are monotone. Finally, we can see that $\hat{\sigma}$ is monotone as follows: If $T \subset$ $\mathscr{L}(\mathscr{X}), \mathscr{M} \in \mathscr{S}_{c ; \infty}(\mathscr{X})$, and $T \mathscr{M} \subseteq \mathscr{M}$ and $T \mathscr{N} \subseteq \mathscr{N}$ (where $\mathscr{M} \dot{+N}=\mathscr{X}$ ), then $\sigma_{T}\left(\left.T\right|_{\mathscr{M}}\right) \subseteq \sigma_{T}(T)$, so that $\hat{\sigma}\left(\left.T\right|_{\mathscr{K}}\right)=\left[\sigma_{T}\left(\left.T\right|_{\mathscr{K}}\right)\right]^{\wedge} \subseteq\left[\sigma_{T}(T)\right]^{\wedge}=\hat{\sigma}(T)$, where denotes polynomially convex hull (recall that $\left(\sigma_{T}\right)^{\wedge}=\hat{\sigma}$ by [15, Theorem 5.2]). A similar argument works for the rationally convex spectrum, using [4, Application 3.9] instead.

PROPOSITION 2.6. Let $\sigma_{1}$ and $\sigma_{2}$ be spectral systems on $\mathscr{X}$, and assume that

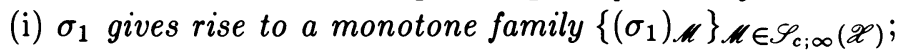

(ii) $\left(\sigma_{1}\right)_{\mathscr{M}} \subseteq\left(\sigma_{\Pi}\right)_{\mathscr{M}}$ for all $\mathscr{M} \in \mathscr{S}_{c ; \infty}(\mathscr{X})$;

(iii) $\sigma_{1}(S, T) \subseteq \sigma_{1}(S) \times \sigma_{1}(T)($ all $(S, T) \subset \mathscr{L}(\mathscr{X}))$;

(iv) $\sigma_{2}(S, T) \subseteq \sigma_{2}(S) \times \sigma_{2}(T)($ all $(S, T) \subset \mathscr{L}(\mathscr{X}))$.

Then, if

(v) isol. $\sigma_{2}(S) \subseteq$ isol. $\sigma_{T}(S)$, we have

$$
P_{S} \sigma_{b ; 1,2}(S, T) \subseteq \sigma_{b ; 1,2}(S)
$$


for all $T$ such that $(S, T) \subset \mathscr{L}(\mathscr{X})$ and $\sigma_{l}(S, T) \cap \sigma_{r}(S, T) \subseteq \sigma_{1}(S, T)$. (Here isol. denotes the set of isolated points.)

REMARK 2.7. Assumptions (i)-(iv) are very mild and satisfied by most spectral systems. The important condition is (v); as we saw before (Example 2.4), not every $\sigma_{2}$ can produce a Browder spectral system satisfying $(*)$. We shall see later that (v) holds in many instances.

ProOF OF PROPOSITION 2.6. Let $(\lambda, \mu) \in \sigma_{b ; 1,2}(S, T)$ and assume that $\lambda \notin$ $\sigma_{b ; 1,2}(S)$, i.e., $\lambda \notin \sigma_{1}(S)$ and $\lambda$ is not a limit point of $\sigma_{2}(S)$. From (iii), $(\lambda, \mu) \notin$ $\sigma_{1}(S, T)$; thus, $(\lambda, \mu) \in \sigma_{2}(S, T)^{\prime}$ and by (iv) we get at once that $\lambda \in \sigma_{2}(S)$. Therefore, $\lambda \in \sigma_{2}(S) \backslash \sigma_{2}(S)^{\prime}$, so that $\lambda$ is in isol. $\sigma_{2}(S) \subseteq$ isol. $\sigma_{T}(S)$. Let $K:=$ $\left(\{\lambda\} \times \sigma_{T}(T)\right) \cap \sigma_{T}(S, T)$ and $L:=\sigma_{T}(S, T) \backslash K$. Clearly $\sigma_{T}(S, T)=K \dot{\cup} L$ and by [15, Theorem 4.9] there exists a decomposition $\mathscr{X}=\mathscr{M} \dot{+} \mathscr{N}$, where $\mathscr{M}$ and $\mathscr{N}$ are invariant for $(S, T)$ and $\sigma_{T}\left(\left.(S, T)\right|_{\mathscr{M}}\right)=K, \sigma_{T}\left(\left.(S, T)\right|_{\mathscr{N}}\right)=L$. It follows that $\sigma_{T}\left(\left.S\right|_{\mathscr{M}}\right)=\{\lambda\}$, so that $\sigma_{\Pi}\left(\left.S\right|_{\mathscr{M}}\right)=\{\lambda\}$. If $\mathscr{M}$ is infinite dimensional, we have $\mathscr{M} \in$ $\mathscr{S}_{c ; \infty}(\mathscr{X})$ and $\sigma_{1}\left(\left.S\right|_{\mathscr{M}}\right)=\{\lambda\}$ (since by (ii), $\sigma_{1}\left(\left.S\right|_{\mathscr{M}}\right) \subseteq\{\lambda\}$ and also $\left.\sigma_{1}\left(\left.S\right|_{\mathscr{M}}\right) \neq \varnothing\right)$. Moreover, $\sigma_{1}(S \mid \mathscr{M}) \subseteq \sigma_{1}(S)$ by monotonicity and thus $\lambda \in \sigma_{1}(S)$, a contradiction. Therefore $\mathscr{M}$ must be finite dimensional. Then $\left\{\nu:(\lambda, \nu) \in \sigma_{T}(S, T)\right\}$ is finite, so that $(\lambda, \mu) \in$ isol. $\sigma_{T}(S, T)$. By another application of [15, Theorem 4.9], $(\lambda, \mu) \in$ $\sigma_{l}(S, T) \cap \sigma_{r}(S, T)$ (see the end of the proof of Theorem 2.11 below) and thus $(\lambda, \mu) \in \sigma_{1}(S, T)$. Therefore, $\lambda \in \sigma_{1}(S)$ (by (iii)), a contradiction. The proof of the proposition is now complete.

THEOREM 2.8. Let $\sigma_{1}$ and $\sigma_{2}$ be two spectral systems possessing the projection property. Assume that $\sigma_{1}$ satisfies (i) and (ii) of Proposition 2.6. Then, if $S \subset$ $\mathscr{L}(\mathscr{X})$ and isol. $\sigma_{2}(S) \subseteq$ isol. $\sigma_{T}(S)$, we have

$$
P_{S} \sigma_{b ; 1,2}(S, T)=\sigma_{b ; 1,2}(S)
$$

for all $T$ such that $(S, T) \subset \mathscr{L}(\mathscr{X})$.

ProOF. Combine Proposition 2.2 and Proposition 2.6 (with its proof).

COROLLARY 2.9. Let $\sigma_{1}$ be a spectral system possessing the projection property and satisfying (i) and (ii) of Proposition 2.6. Then $\sigma_{b ; 1, T}:=\sigma_{1} \cup \sigma_{T}^{\prime}$ possesses the projection property.

COROLlaRY 2.10. $\sigma_{b}:=\sigma_{T e} \cup \sigma_{T}^{\prime}$ possesses the projection property.

We shall see now that $\sigma_{2}$ can be quite general and still satisfy (iv) and (v) of Proposition 2.6.

THEOREM 2.11. Let $S \subset \mathscr{L}(\mathscr{X})$ and let $\mathscr{B}$ be a commutative unital subalgebra of $\mathscr{L}(\mathscr{X})$ containing $S$. Let $\lambda$ be an isolated point of $\sigma_{\mathscr{B}}(S)$. Then $\lambda$ is an isolated point of $\sigma_{T}(S)$ and, a fortiori, an isolated point of $\sigma_{l}(S) \cap \sigma_{r}(S)$.

ProOF. We know that $\sigma_{\mathscr{B}}(S)=\{\lambda\} \dot{\cup} K$, where $K$ is compact. Assume that $\lambda \notin \sigma_{T}(S)$; since $\sigma_{T}(S) \subseteq \sigma_{\mathscr{B}}(S)$ we must have $\sigma_{T}(S) \subseteq K$. Let $P=f(S) \in \mathscr{B}$ be the idempotent associated with $\lambda$ constructed via the Shilov-Arens-CalderónWaelbroeck functional calculus. Then $\sigma_{\mathscr{B}}(P)=f\left(\sigma_{\mathscr{B}}(S)\right)=\{0,1\}$, while $\sigma_{T}(P)=$ $f\left(\sigma_{T}(S)\right)=\{0\}$. Since $\sigma_{\mathscr{B}}(P) \subset \hat{\sigma}(P)$ and $\hat{\sigma}(P)=\left[\sigma_{T}(P)\right\}$, we get a contradiction. Thus, $\lambda \in \sigma_{T}(S)$. The statement about $\sigma_{l} \cap \sigma_{r}$ follows from the fact that if $\sigma_{T}(S)=\{\lambda\} \dot{\cup} L(L \subseteq K)$, then $\mathscr{X}=\mathscr{M} \dot{+} \mathscr{N}$ with $\sigma_{T}\left(\left.S\right|_{\mathscr{M}}\right)=\{\lambda\}[\mathbf{1 5}$, Theorem 
4.9]. Then $\sigma_{l}\left(\left.S\right|_{\mathscr{M}}\right)=\{\lambda\}=\sigma_{r}\left(\left.S\right|_{\mathscr{M}}\right)$, so that $\lambda$ is an isolated point of $\sigma_{l}(S) \cap \sigma_{r}(S)$.

REMARK 2.12. If $\lambda$ is an isolated point of $\sigma_{\mathscr{B}}(S)$, the idempotent $P$ in Theorem 2.11 splits the algebra as $\mathscr{B}=P \mathscr{B}+(I-P) \mathscr{B}$ and, by $[17,20.2], P$ also splits the maximal ideal space of $\mathscr{B}$ as $M_{\mathscr{B}}=F_{1} \dot{\cup} F_{2}$, where $F_{1}:=\left\{\varphi \in M_{\mathscr{B}}: \varphi(P)=1\right\}$ and $F_{2}:=\left\{\varphi \in M_{\mathscr{B}}: \varphi(P)=0\right\}$. The proof of Theorem 2.11 also shows that $M_{\sigma_{T}}$ (the compact nonempty subset of $M_{\mathscr{B}}$ associated with $\sigma_{T}$ (see $\S 3$ below)) is such that $M_{\sigma_{T}} \cap F_{1} \neq \varnothing$ (otherwise $\varphi(P)=0$ for all $\varphi \in M_{\sigma_{T}}$, so that $\sigma_{T}(P)=$ $\left.\Gamma(P)\left(M_{\sigma_{T}}\right)=\{0\}\right)$.

3. Applications. Let $\tilde{\sigma}$ be a spectral system on $\mathscr{X}$ possessing the projection property. Let $\mathscr{B}$ be a commutative unital Banach subalgebra of $\mathscr{L}(\mathscr{X})$ and assume that $\tilde{\sigma}(S) \subseteq \sigma_{\mathscr{B}}(S)$ for all $S \subset \mathscr{B}$. Then $\tilde{\sigma}$ admits a functional representation as follows $([\mathbf{1 8}]$; see also $[4, \S 3])$ :

There exists a compact nonempty subset of $M_{\mathscr{B}}, M_{\tilde{\sigma}}$, such that

$$
\tilde{\sigma}(S)=\Gamma(S)\left(M_{\tilde{\sigma}}\right) \quad(\text { all } S \subset \mathscr{B})
$$

where $\Gamma: \mathscr{B} \rightarrow C\left(M_{\mathscr{B}}\right)$ is the Gelfand transform. Moreover, $M_{\tilde{\sigma}}$ is unique relative to $(* *)$. If $f$ is a (vector-valued) function analytic in a neighborhood of $\sigma_{\mathscr{B}}(S)$ (so that $f(S)$ is a well-defined tuple in $\mathscr{B})$, then

$$
\begin{aligned}
\tilde{\sigma}(f(S)) & =\Gamma(f(S))\left(M_{\tilde{\sigma}}\right)=(f \circ \Gamma(S))\left(M_{\tilde{\sigma}}\right) \\
& =f\left[\Gamma(S)\left(M_{\tilde{\sigma}}\right)\right]=f(\tilde{\sigma}(S)),
\end{aligned}
$$

i.e., $\tilde{\sigma}$ has the spectral mapping property for analytic functions. If $f$ is analytic only in a neighborhood of $\sigma_{T}(S), f(S)$ still makes sense (as an operator on $\mathscr{Z}$ which belongs to the double commutant of $S$ ) but it may not belong to $\mathscr{B}$. If it does, then $\tilde{\sigma}(f(S))=f(\tilde{\sigma}(S))$ again. If $\tilde{\sigma}(S) \subseteq \sigma_{T}(S)$, we can use $\mathscr{B}=(S)^{\prime \prime}$ to see that the spectral mapping property holds for all (vector-valued) functions analytic in neighborhoods of $\sigma_{T}(S)$.

THEOREM 3.1. Let $\sigma_{1}$ and $\sigma_{2}$ be spectral systems on $\mathscr{X}$ such that $\sigma_{b ; 1,2}$ possesses the projection property. Let $\mathscr{B}$ be a commutative unital Banach subalgebra of $\mathscr{L}(\mathscr{X})$, let $S \subset \mathscr{B}$ and assume that $\sigma_{b ; 1,2}(S) \subseteq \sigma_{\mathscr{B}}(S)$. Then for any (vectorvalued) function $f$ analytic in a neighborhood of $\sigma_{\mathscr{B}}(S)$ one has

$$
\sigma_{b ; 1,2}(f(S))=f\left(\sigma_{b ; 1,2}(S)\right) .
$$

If, in addition, $\sigma_{b ; 1,2}(S) \subseteq \sigma_{T}(S)$ then the spectral mapping property holds for all (vector-valued) functions analytic in neighborhoods of $\sigma_{T}(S)$.

PROOF. Immediate from the preceding remarks.

COROLlARY 3.2. Let $S \subset \mathscr{L}(\mathscr{X})$ and let $f$ be a (vector-valued) function analytic in a neighborhood of $\sigma_{T}(S)$. Then

$$
\sigma_{b}(f(S))=f\left(\sigma_{b}(S)\right)
$$

where $\sigma_{b}:=\sigma_{T e} \cup \sigma_{T}^{\prime}$.

Thus, Gramsch and Lay's result [9, Theorem 4] extends to $n$ variables.

We shall conclude with a calculation of Browder spectra for $n$-tuples of tensor products. 
In [5], it was shown that

$$
\sigma_{T e}(S \otimes I, I \otimes T)=\left[\sigma_{T e}(S) \times \sigma_{T}(T)\right] \cup\left[\sigma_{T}(S) \times \sigma_{T e}(T)\right],
$$

where $S, T \subset \mathscr{L}(\mathscr{H}), \mathscr{H}$ a Hilbert space. Since

$$
\sigma_{T}(S \otimes I, I \otimes T)=\sigma_{T}(S) \times \sigma_{T}(T)
$$

[2], we immediately get the following fact:

$(* * *) \quad \sigma_{b}(S \otimes I, I \otimes T)=\left[\sigma_{b}(S) \times \sigma_{T}(T)\right] \cup\left[\sigma_{T}(S) \times \sigma_{b}(T)\right]$

(as before, $\sigma_{b}=\sigma_{T e} \cup \sigma_{T}^{\prime}$ ). $(* * *$ ) generalizes [14, Theorem 3 and 6, Theorem 7]. A similar formula can be obtained for certain tensor products acting on Banach spaces using the results in [7].

Since $\sigma_{b}$ satisfies the spectral mapping theorem for functions analytic in neighborhoods of $\sigma_{T}$, we obtain

$$
\sigma_{b}(f(S \otimes I, I \otimes T))=f\left(\sigma_{b}(S) \times \sigma_{T}(T)\right) \cup f\left(\sigma_{T}(S) \times \sigma_{b}(T)\right),
$$

which extends [14, Theorem 2].

Concerning $\sigma_{e}^{\mathrm{dc}}(S \otimes I, I \otimes T)$ ( $\sigma_{e}^{\mathrm{dc}}$ denotes essential double commutant spectrum), it is known that

$$
\left[\sigma_{e}(S) \times \sigma(T)\right] \times\left[\sigma(S) \times \sigma_{e}(T)\right] \subseteq \sigma_{e}^{\mathrm{dc}}(S \otimes I, I \otimes T)
$$

$(S, T$ operators on $\mathscr{H}$; see $[\mathbf{1 2}])$, and that

$$
\sigma_{b}(S \otimes I, I \otimes T)=\sigma_{b}^{\mathrm{dc}}(S \otimes I, I \otimes T)
$$

[6], which seems to indicate that the previous containment is always an equality, although no proof (or counterexample) has yet been found.

\section{BIBLIOGRAPHY}

1. J. J. Buoni, A. T. Dash, and B. L. Wadhwa, Joint Browder spectrum, Pacific J. Math. 94 (1981), 259-263.

2. Z. Ceauşescu and F.-H. Vasilescu, Tensor products and the joint spectrum in Hilbert spaces, Proc. Amer. Math. Soc. 72 (1978), 505-508.

3. R. E. Curto, Connections between Harte and Taylor spectra, Rev. Roumaine Math. Pures Appl. 31 (1986), 203-215.

4. __ Applications of several complex variables to multiparameter spectral theory, Survey of Recent Results in Operator Theory, vol. II, J. B. Conway (editor), Longman (to appear).

5. R. E. Curto and L. A. Fialkow, The spectral picture of $\left(L_{A}, R_{B}\right)$, J. Funct. Anal. 71 (1987), 371-392.

6. A. T. Dash, Joint Browder spectra and tensor products, Bull. Austral. Math. Soc. 32 (1985), 119-128.

7. J. Eschmeier, Tensor products and elementary operators, preprint, 1986.

8. L. A. Fialkow, Spectral properties of elementary operators. II, Trans. Amer. Math. Soc. 290 (1985), 415-429.

9. B. Gramsch and D. Lay, Spectral mapping theorems for essential spectra, Math. Ann. 192 (1971), 17-32.

10. R. E. Harte, Tensor products, multiplication operators and the spectral mapping theorem, Proc. Roy. Irish Acad. 73A (1973), 285-302.

11. M. Putinar, Functional calculus and the Gelfand transformation, Studia Math. 79 (1984), 83 86.

12. M. Schecter and M. Snow, The Fredholm spectrum on tensor products, Proc. Roy. Irish Acad. 75A (1975), 121-127. 
13. Z. Słodkowski, An infinite family of joint spectra, Studia Math. 61 (1977), 239-255.

14. M. Snow, A joint Browder essential spectrum, Proc. Roy. Irish Acad. 75A (1975), 129-131.

15. J. L. Taylor, A joint spectrum for several commuting operators, J. Funct. Anal. 6 (1970), 172191.

16. $\ldots$, The analytic functional calculus for several commuting operators, Acta Math. 125 (1970), 1-38.

17. W. Żelazko, Banach algebras, Elsevier, New York, 1973.

18. __ An axiomatic approach to joint spectra. I, Studia Math. 64 (1979), 250-261.

Department of Mathematics, University of IOWA, IOWA City, IOWA 52242

DEPARTMENT OF MATHEMATICS AND Statistics, UNiVERSity OF GUELPh, GUELPH, ONTARIO, CANADA N1G 2W1 